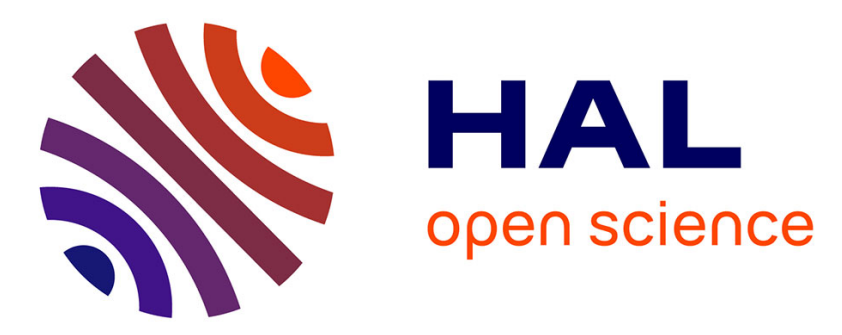

\title{
Wissen, Lieben - und Schreiben Phantastik und Skepsis im Briefwechsel Chamissos mit seiner Frau aus dem Sommer 1823
}

Anne Baillot

\section{- To cite this version:}

Anne Baillot. Wissen, Lieben - und Schreiben Phantastik und Skepsis im Briefwechsel Chamissos mit seiner Frau aus dem Sommer 1823. Phantastik und Skepsis Die Lebens- und Schreibwelten des Adelbert von Chamisso, May 2013, Berlin, Germany. p. 351-367. halshs-01132315

\section{HAL Id: halshs-01132315 \\ https://shs.hal.science/halshs-01132315}

Submitted on 17 Mar 2015

HAL is a multi-disciplinary open access archive for the deposit and dissemination of scientific research documents, whether they are published or not. The documents may come from teaching and research institutions in France or abroad, or from public or private research centers.
L'archive ouverte pluridisciplinaire HAL, est destinée au dépôt et à la diffusion de documents scientifiques de niveau recherche, publiés ou non, émanant des établissements d'enseignement et de recherche français ou étrangers, des laboratoires publics ou privés.

\section{(c)(1)}

Distributed under a Creative Commons Attribution| 4.0 International License 


\section{Wissen, Lieben - und Schreiben \\ Phantastik und Skepsis im Briefwechsel Chamissos mit seiner Frau aus dem Sommer 1823}

Anne Baillot, November 2014

Im Sommer 1823 unternahm Adelbert von Chamisso eine Beobachtungseise nach Greifswald und Rügen und ließ seine Familie währenddessen in Berlin zurück. Der Briefwechsel, den er zwischen dem 10. Juni und 17. Juli mit seiner Ehefrau Antonie führte, beinhaltet v. a. Reflexionen zum naturwissenschaftlichen Zweck der Reise. Er befasst sich aber auch mit dem Schreibprozess im Dialog mit der geliebten Adressatin. Damit knüpfen diese Briefe sowohl an die Jugendbriefe Chamissos mit seinen damaligen Freunden an und an die Weltreisebriefe. Im Korpus an Reisebriefen, worum es hier gehen soll, liegt aber - verglichen mit der Weltreise - die Fremdheitserfahrung nicht in der Auseinandersetzung mit fremden Kontinenten, sondern mit dem älter gewordenen Ich.

Um den Bogen zwischen Jugend und Reife geht es hier, und um diesen zu spannen, ist der erste Teil dieses Aufsatzes dem Briefwechsel Chamissos mit seinem Freund Louis de La Foye aus den Jahren 1804 bis 1812 gewidmet, einer Zeit, in der der Dichter viel umherreiste, bis er sich in Berlin endgültig niederließ. Insbesondere um die Thematisierung des Verhältnisses von (Natur)wissenschaft und Dichtung soll es in diesem Korpus gehen, ein Themenkomplex, das im Anschluss im Kontext des Briefwechsels mit der Ehefrau Antonie aus dem Sommer 1823 unter die Lupe genommen wird. ${ }^{1}$ In beiden Briefkorpora kristallisiert das Motiv des Reisens die Inszenierung von Entfernung und deren Überwindung durch den Akt des Schreibens. So vermischt sich die Reise als naturwissenschaftliche Erkundung mit Überlegungen zur Gestaltung der Liebesbeziehung und der Schreiberidentität. Das Zusammenspiel dieser Elemente im Kontext des hier betrachteten Korpus wird untersucht, ehe in einem letzten Schritt der Analyse auf die Ergiebigkeit des Briefwechsels mit Antonie für das schriftstellerische Wirken Chamissos eingegangen wird.

\section{1. „Wir brauchen nur eine Stube und ein Bett ${ }{ }^{2}$ - die Briefe der frühen Jahre an Louis de La Foye}

In der Zeit zwischen 1804 und 1812 war Chamisso ständig auf dem Sprung, noch wusste er nicht, wo er sich niederlassen sollte. Von 1798 bis 1806 diente er im Regiment von Götze, zuerst als Fähnrich und seit 1801 als Leutnant. Ab Dezember 1806 hielt er sich in Paris auf, im Sommer 1807 bei den Geschwistern in Vertus und Troyes, kehrte nach Berlin zurück, ehe er sich 1810 wieder nach Paris begab. Nach nur wenigen Monaten in der französischen Hauptstadt schloss er sich Ende September 1810 Mme de Staël in Chaumont an, auf deren Vermittlung er den Winter in Napoléon-Vendée verbrachte. 1811 stieß Chamisso erneut zu jenem Kreis um Mme de Staël, zunächst in Coppet und

\footnotetext{
${ }^{1}$ Beide Korpora wurden zwischen 2011 und 2014 im Rahmen von BA-, MA- und Hausarbeiten von Studenten der Europäischen und Deutschen Literatur der Humboldt-Universität zu Berlin neu transkribiert und ediert. Sie sind in der digitalen Edition Briefe und Texte aus dem intellektuellen Berlin um 1800 (http://tei.ibi.huberlin.de/berliner-intellektuelle/; Stand 13. November 2014) einsehbar. Ich bedanke mich bei Lena Ebert und Henrike Bobzien dafür, dass sie es mir ermöglicht haben, diese Untersuchung nicht auf der Grundlage von redigierten Editionen, sondern auf ihrer Arbeit mit den Originalhandschriften basierend zu konzipieren. Ich werde hier nicht auf die Überlieferungsgeschichte der Korpora eingehen, es sei nur darauf hingewiesen, dass, im Falle des Briefwechsels mit de La Foye beinahe ausschließlich die Briefe von Chamisso und kaum Gegenbriefe vorliegen, während die Briefe Antonies so gut erhalten sind wie diejenigen Chamissos und der briefliche Dialog sich durchgehend rekonstruieren lässt.

${ }^{2}$ Brief an de La Foye vom 16. Januar 1807 (ab hier, wenn nicht anders angegeben, gilt jeder Verweis auf Briefe und Texte aus dem intellektuellen Berlin, wie Anm. 1, Stand 13. November 2014).
} 
später in Genf, von wo aus er mehrere Alpenexkursionen unternahm. 1812 begab er sich dann endgültig nach Berlin.

Diese extrem mobile Situation gab Anlass zu ausgeprägten existentiellen Betrachtungen, die sowohl Weltskepsis als auch phantastische Selbstinszenierungen Ausdruck verleihen. Im Briefwechsel mit den engsten Freunden kommen diese variierenden Pole am deutlichsten zum Ausdruck, so anhand der Briefe an den Freund de La Foye, der in vielfacher Hinsicht in Chamissos Briefen als Reflexionsfläche fungierte. Dieses Identifikationsphänomen hat gute Gründe, springen doch die Schicksalsparallelen beider Emigrationskinder sofort ins Auge. Ebendiese Parallelen wurden nicht zuletzt von der (allerdings verhältnismäßig spärlichen) Forschung stets in den Vordergrund gestellt. ${ }^{3}$ Kennengelernt haben sich Adelbert von Chamisso und Louis de La Foye 1803, vermutlich über Julius Eduard Hitzig ${ }^{4}$, Chamissos engen Freund, literarischen Berater, künftigen Verleger und Schwiegervater ${ }^{5}$. Zusammen mit Julius Eduard Hitzig spielen in den Briefen an de La Foye alle Freunde aus dem Nordsternbund ${ }^{6}$ eine wichtige Rolle. Dieser literarischen Vereinigung, die von 1803 bis 1806 bestand, gehörten neben Chamisso, de La Foye, Hitzig und Varnhagen u. a. auch Neumann, Fouqué, Robert, Theremin an. In den von Chamisso und Varnhagen 1804 bis 1806 herausgegebenen Musenalmanachen ${ }^{7}$ veröffentlichten die Mitglieder des Nordsternbundes ihre lyrischen Werke. Nachrichten der Nordsternbund-Freunde und Bruderschaftsbeteuerungen gehörten zu den strukturierenden Topoi der Briefe Chamissos an de La Foye, so etwa die häufig verwendete Anrede "Adelf" (Bruder).

Eine weitere gemeinsame Erfahrung prägt den Briefwechsel zwischen Chamisso und de La Foye der Jahre 1804-1812 in besonderem Maße: die Erlebnisse in der preußischen Armee. Ab Ende 1799 war de La Foye Offiziersanwärter im Regiment Winning, kehrte aber 1804 nach dem Tod seines Vaters nach Frankreich zurück. Chamisso war es, der sich um de La Foyes Ausscheiden aus der Armee kümmerte. ${ }^{8}$ Bei Chamisso hinterließ die Zeit beim preußischen Militär tiefe Spuren, die nicht ohne Bezug zu seiner naturwissenschaftlichen Laufbahn standen. Im Herbst 1812, endlich in Berlin als Student der Medizin immatrikuliert, berichtete er etwa:

Der Wissenschaft will ich durch Beobachtung und Erfahrung, sammeln und vergleichen mich nähern - vergessen hab ich schon daß ich je ein Sonnet geschrieben. - Gott verzeihe mir meine Sünden - Der alte freundliche Knape, trocken wie seine Lehre von den trockenen Knochen, frug mich letzhin auf dem anatomischen Saale wo er das Prepariren dirigirte, - auf welcher Universität ich früher studirt - Im Regiment von Götze gab ich inm zu Antwort $-^{9}$

\footnotetext{
${ }^{3}$ Dufraisse 1995, S. 155-166; Ders. 1982, S. 63-90.

${ }^{4}$ Hierzu vgl. Busch 2014.

${ }^{5}$ Hitzig war Ziehvater der 1800 geborenen Antonie Piaste, die Chamisso 1819 heiratete.

${ }^{6}$ Busch (u. a.) 2014.

${ }^{7}$ Chamisso / Varnhagen (Hg.): Musenalmanach auf das Jahr 1804. Leipzig 1804; Dies.: Musenalmanach auf das Jahr 1805. Zweiter Jahrgang. Berlin 1805; Dies.: Musenalmanach auf das Jahr 1806. Dritter Jahrgang. Berlin 1806.

8 "Deinen Abschied habe ich diese Tage erhalten, nach langen langsamen Bemügungen, die Hunde haben es übel genomen und sie haben darüber schwer geseufzet daß du das Blat geschnitten hattest, und so weiter [...]" (Brief an de La Foye vom 1. März 1805). Vgl. schon im Brief an de La Foye vom 20. September 1804: „Vergiß nicht daß du noch um ein Abschied anzuhalten hast."

${ }^{9}$ Brief an de La Foye vom Herbst 1812. Auch Chamisso, wie andere Emigranten, und darin auch den preußischen Hugenottenkindern nahe, bekennt sich zum preußischen Patriotismus (vgl. Brief von 1813, wo er von der Entstehung des Schlemihl berichtet), was in den napoleonischen Kriegen dazu führte, dass französischsprachige Soldaten einander bekämpften. Im Brief an de La Foye vom Winter 1814 schreibt er
} 
Damit wird eine gerade Linie zwischen Militär und Wissenschaft gezogen, in die die dichterische Karriere nolens volens eingebettet wird. Chamisso gibt an, er hätte "vergessen“, „je ein Sonnet geschrieben" zu haben, so wie er an anderer Stelle in einem vergleichbaren Gestus behauptete, sich vom "Dichtisiren“ seiner frühen Jahre zu distanzieren. ${ }^{10}$ Doch die Erwähnung des Vergessens ruft gezwungenermaßen die Erinnerung hervor. Das Dichten steht hier wie eine Klammer zwischen militärischer und naturwissenschaftlicher Selbstbestimmung - aber als eine Klammer, die sich nicht ausklammern lässt.

1804 siedelte sich de La Foye infolge der napoleonischen Bemühungen, die emigrierten Adelsfamilien nach Frankreich zurückzuholen und des Todes seines Vaters (Mai 1804) in der Normandie an, während Chamisso mehrere Jahre lang keinen festen Wohnsitz hatte. Durch die Freunde des Nordsternbundes animiert und sich dem Projekt des grünen Almanachs ${ }^{11}$ leidenschaftlich widmend, beschäftigte sich Chamisso in dieser Zeit mit Feinheiten der deutschen Metrik. ${ }^{12}$ Gleichzeitig spekulierte er auf ein gemeinsames Leben mit de La Foye:

[D]as dünkt mich wie unser alte Traum. Wir brauchen nur eine Stube und ein Bett, bist du auf deinem Bureau, quäl ich mich und die Deutsche Sprache redlich daheim daß Verse draus werden [.....$^{13}$

Den Traum eines Dichterlebens gab Chamisso jedoch auf, wie es der spätere Briefwechsel zeigt. Im August 1811 hieß es aus Anlass des Kennenlernens von Jean Charles Léonard Simonde de Sismondi, dieser sei „ohne das was den Dichter macht“, was zu einer Selbstverortung Anlass gab:

Solches erstirbt auch mehr und mehr in mir, es war wirklich eine Zeit wo wir die Ruthe verdienten. - was ich itzt verdiene - ich weiß es wahrlich nicht doch auf jeden Fall wenig genug. ${ }^{14}$

explizit: „Nie hab ich mehr unlust an der Politische und mehr ekel gegen Frankreich empfunden als eben jezt. ich schätze mich sehr glücklich nicht da zu sein und begehre gar nichts". Bereits von Coppet aus hatte er sich 1811-1812 nach seinem "Norden“ gesehnt (im Brief von Mai oder Juni 1811 erwähnt er das Vorhaben, in Deutschland zu studieren). Die Assoziation zwischen Norddeutschland und dem Nordsternbund wird im Brief vom 1. März 1805 explizit: „Auch die Beibehaltung der Pfeife lob' ich, sie ist dir jetzt die Frucht des Vaterlandes [...] müssen wir doch nach dem Aufgang die Augen werfen, dem Lande der Erlosung unserem Deutschlande, und wieder uns sehnen, wie nach dem Ewigkeits Blicke des Nordes Sternes selber schauen, den deutschen sind wir doch".

${ }^{10} \mathrm{Vgl}$. auch den Brief vom 12. August 1805 an Varnhagen und Neumann: „O Freunde, lasset uns nicht [...] die Zeit, mit Bemühungen des Dichtisiren zerfetzen! und Machwerke doch zum öftern nur machen. [...] Das lege ich Euch ans Herz, für mein Theil will ich nicht dichten wollen." (Palm 1864, S. 81).

${ }^{11}$ Aufgrund der grünen Einbandfarbe wurden die Musenalmanache auch als „Grüne" bezeichnet.

${ }^{12}$ Ende Oktober 1804 schreibt Chamisso an de La Foye: „ich werde nichts schreiben, das nicht probe halte", und kommt im Brief an de La Foye zwischen Mitte März und Anfang April 1805 auf metrische Feinheiten zu sprechen, ohne sich und Varnhagen jedoch mit dem „Meister[] der Versifikazions Kunst Voss vergleichen“ zu wollen.

${ }^{13}$ Brief an de La Foye vom 16. Januar 1807. Mit dem „alten Traum“ meint er sicherlich den bereits im Brief vom 15. August 1804 erwähnten: „Solltest du, nicht Offizier mehr, wiederum nach unserm Norden deine Schritte leiten, so hast du auf dieses zu rechnen: 1. die Hälfte meines Bettes (so lang ich unverheiratet bin) meines Zimmers, meines Lichtes, meiner Heizung. -2 auch wenn es sein soll die kleinere Hälfte meines täglichen Brotes da ich den grosseren Apetit habe [...]“. Also 1804 ein Berliner, 1807 ein Pariser Traum.

${ }^{14}$ Brief an de La Foye vom 16. August 1811. Die Ruthe kommt in Bezug auf Kindererziehung im Brief Chamissos an Antonie vom 21. Juni 1823 wieder vor: „wird er[= der Sohn] beleidigend unartig - die Ruthe, aber ohne viel davon zu sprechen: Nie ohne Noth, nie ohne Nachdruck. [...] aber die Poesie, der Scherz, die Fable, wovon so ein junges Leben vollsteckt, ja nicht für Lüge ansehen." 
Die Zeit in Genf und Coppet (von 1811 bis 1812) als Gast Germaine de Staëls empfand er als eine unglückliche Lebensphase. Die Briefe an de La Foye dokumentieren insbesondere Selbstzweifel und ein Gefühl der Unzugehörigkeit, das teilweise in Selbst-, teilweise in Weltverachtung mündete.

Der Ausbruch des Krieges hinderte ihn daran, Coppet rechtzeitig zu verlassen, ${ }^{15}$ um sich seinem Wunsch gemäß zum Sommersemester 1811 an der Berliner Universität zu immatrikulieren. ${ }^{16} \mathrm{Im}$ Brief vom Winter 1811 ging er eindrücklich darauf ein, dass er sich fehl am Platz fühle, dabei seine Unzufriedenheit mit der Coppet'schen „schönen“ Gesellschaft andeutend:

Ich spiele den Brumbären in dieser glanzenden rauschenden feinen Welt wo lerm für musik, Eleganz für Gehalt, und Maul Tasche wesen für alle geraubte Freiheit dienen soll. ${ }^{17}$

Die Kritik am sozialen Kerker wurde zu einem Motiv des in sich verfaulenden Körpers weitergesponnen:

ich bin müde über alle massen, ich habe keinen willen mehr ich sehe es noch mit an, daß mir die Beine vom Leibe weg abfaulen. [...] Den Sommer werd ich wohl nicht einmal dazu kommen, einen Spaziergang ins Gebürg zu unter nehmen, ich seh' es kommen. ich weiß schon wie das alles hier geht. Ich bin mit W.[ilhelm] A.[ugust] Sch.[legel] sehr mißvergnügt wenn wir zusammen im selben Loche eingesperrt sind weiß ich eben nicht wie es gehen wird $[\ldots]^{18}$

Zwischen Einkerkerung in sich selbst und in der Welt einerseits und Freiheit der naturwissenschaftlichen Selbstbildung andererseits sind Reisen (und seien es einfache Wanderungen) als Schlüsselerlebnisse zu verorten. Chamisso füllte seine Flucht aus einer als mangelhaft empfundenen sozialen Identität mit Topoi des Reisens. Seit Herbst 1811 widmete er sich der Lektüre von Reiseliteratur ${ }^{19}$ und bereitete Exkursionen in die Alpen vor:

auch will ich itzt, als mein eigener Herr, bei meinem, auf deinem anstoßen unter nommenen, botanischen Studium, hier den hohen Sommer erwarten, sodann ins gebürg, und nach vollbrachter Wahlfahrt, mißgönnen es da nicht die Zeit umstände, leise nach meinem Norden abrutschen. [...] ich kenne schon nach delamarck und De Candolle an ein Paar Hundert Blumen [...] die stillen unschuldigen Blumen, unterhalten mich wirklich besser und vertraulicher als Menschen $[\ldots]^{20}$

So wurden die botanischen Streifzüge durch die Alpen ${ }^{21}$ Momente der Selbstfindung, die Hand in Hand mit einer Neuentdeckung der Natur gingen - nicht zuletzt auf den Impuls de La Foyes, der seinem Freund die Botanik ans Herz legte.22 Die Schreibbewegung ist dabei eine doppelte: Es geht sowohl um eine weltliche Abgrenzung von einer als künstlich empfundenen Geselligkeit als auch um die Verabschiedung eines als ebenso künstlich betrachteten Naturlobs in Versen. Die sich

\footnotetext{
${ }^{15}$ Vgl. Brief an de La Foye vom Herbst oder Winter 1811: „Ich mache mir selber weiß daß eben die Gründe die man mir zu gehen gegeben, mich zu bleiben bestimmt haben; die furcht die man mir nemlich in die Glieder jagen wollen als wäre der Standpunkt gefährlich - es ist im Grunde nichts daran - ich bin nur aus mangel an Thatkraft geblieben, aus furcht vor der Kälte, vor dem Kriege im Norden“; Brief an de La Foye von Anfang 1812: „ich habe den Winter auf die alte weise verbracht, am alten Joche gottweißwie gebunden.“

${ }^{16} \mathrm{Vgl}$. Brief an de La Foye vom 16. August 1811.

${ }^{17}$ Brief an de La Foye vom Herbst oder Winter 1811.

${ }^{18}$ Brief an de La Foye aus Genf, Anfang 1812.

${ }^{19} \mathrm{Vgl}$. Brief an de La Foye vom Herbst oder Winter 1811.

${ }^{20}$ Brief an de La Foye von Genf oder Coppet, Frühling 1812.

${ }^{21}$ Insbesondere in Begleitung Auguste de Staëls; vgl. Brief an de La Foye vom Juni oder Juli 1812: „Morgen gehn wir zum vierten Mal in's Gebürg".

${ }^{22}$ Vgl. Schmid 1942, S. 14ff. sowie Hitzig 1839, S. 244, FN und Br. 136.
} 
anschließende Reise nach Berlin, zum lang ersehnten Norden, wurde ihrerseits als Heimatfindung in Szene gesetzt: „ich ergreife den wanderstaab und laufe nach berlin, aus mir kann nimmermehr ein Pariser werden “23. Einmal in Berlin angekommen (Herbst 1812), fielen Identität mit Beschäftigung und Lebensort erstmalig zusammen und wurden mit der entsprechenden Entschiedenheit auch in Kombination miteinander verkündet:

Ich lebe einmal mit mir und der Welt in Eintracht, und aus der Lüge heraus. Ich habe verständig gewählt und ausgeführt, und bin einmal was ich heiße und heisse was ich bin - Dies ist Studioseo Medicinae der Universität zu Berlin. ${ }^{24}$

Damit kann der Bogen gespannt werden von der anfänglichen militärischen Fremdbestimmung bis zum festen, selbst bestimmten Lebensort, von 1804 bis 1812, oder vielmehr der Kreis von Berlin nach Berlin, nachverfolgt. Die Briefe an de La Foye aus dieser Zeit zeigen Chamissos Wende von der Dichtung zu den Naturwissenschaften als das Ergebnis einer Identitätssuche, die sich durch eine Reihe von Orts- und Tätigkeitswechseln vollzog. Die immer stärker werdende Geselligkeitskritik veranschaulicht darüber hinaus, wie die Beziehung zu ausgewählten Briefpartnern für die Konstruktion dieses Selbstverständnisses des Dichters und Botanikers von zentraler Bedeutung war. $^{25}$

1812 war Chamisso jedoch bei Weitem noch nicht am Ende seiner Selbstsuche. Auf das Studium folgte eine Weltreise von 1815 bis 1819, dann eine Anstellung am Königlichen Herbarium, 1819 die Eheschließung mit Antonie. 1820 und 1822 wurden die Söhne Ernst und Max geboren.

Mitten in dieser kleinbürgerlichen Idylle brach Chamisso im Sommer 1823 nach Greifswald auf, um Barometermessungen vorzunehmen.

\section{2. „[J]ung wieder werden in $\mathrm{Dir}^{\prime 26}$ : die Briefe Chamissos an seine Frau}

Die Tatsache, dass er die Strecke zwischen Berlin und Greifswald laufend zurückslegen wollte, ließ sein familiäres Umfeld stutzen. ${ }^{27}$ Möglicherweise motivierte ihn die Erinnerung an die als befreiend empfundenen Alpenexkursionen. Gleich im ersten Brief zeigte sich allerdings eine gewisse Skepsis:

Ich habe mein Barometer so weit wohlbehalten gebracht, aber langweilig, langweilig und abermals langweilig ist eine solche Reise. ${ }^{28}$

Es dauerte nicht lange, bis er darauf verzichtete, sein Barometer selbst zu tragen. Er holte sich streckenweise Unterstützung und kommentierte dies folgendermaßen:

\footnotetext{
${ }^{23}$ Brief an de La Foye von Juni oder Juli 1812. Vgl. schon im Brief an de La Foye vom 16. August 1811: „Ich bin wirklich noch hier, mein Lieber [...]. Es soll Anfangs künftigm Monats los-, und davon gehen. meiner Seits vermutlich füßlings durch die Schweiz und so fort nach Nord deutschland allein [...]"

${ }^{24}$ Brief an de La Foye vom Herbst 1812.

${ }^{25} \mathrm{Vgl}$. die Schilderung seines Berliner Lebens ebd.: „Ich habe fast aller schönen Geselligkeit entsagt, und lebe nur mit dem prächtigen geduldigen Hitzig. - ich wohne mit dem guten stachellosen Neumann zusammen, der auch pracktisch geworden [...]. Varnhagen florirt hier [...] er lebt in der vornehmsten Welt, ich in meiner Tabacks- Brennerei und Theatrum anatomium."

${ }^{26}$ Brief an Antonie vom 25. Juni 1823. Die Briefe der Greifswalder Reise werden Ende 2014 im Rahmen der digitalen Edition Briefe und Texte aus dem intellektuellen Berlin um 1800 ediert. Zitiert wird hier nach der Transkription von Henrike Bobzien.

${ }^{27}$ Vgl. z.B. Brief von Antonie an Chamisso vom 14. und 15. Juni 1823: „Nachmittags ging ich mit den Kindern zu Pohls, die Tante nimmt den größten Antheil an Dir und hat so unendlich oft, ihr Mitleid mit Dir ausgesprochen, sie konnte sich über Dein zu Fuß gehen, in der Hitze, gar nicht trösten. Zu Hitzig ging ich erst spät, fand da die ganze Familie, Hehring, Forstner, und Sander. Hitzig grüßt Dich herzlich. Erman, Lichtenstein und Andere sagen Du hättest nicht nöthig gehabt zu Fuß zu gehen.“

${ }^{28}$ Brief an Antonie vom 11. Juni 1823.
} 
[...] es war mir ganz recht. Ich hatte von der Hitze gelitten, mein Pack war mir sehr fatal, mein Ueberrock, meine Stiefel dazu, die zu weit sind und sich schlecht eintreten. ${ }^{29}$

Diese wenigen Attribute (Pack, Überrock, Stiefel) evozieren in auffällig dichter Form zentrale Deklinationen von Chamissos literarischer Persönlichkeit der vorangegangenen zehn Jahre: als Schlemihl-Alter Ego einerseits und als Weltumsegler andererseits. Dennoch ziehen im Hintergrund nicht nur Weltgewandtheit und wissenschaftliche Anerkennung, sondern auch Selbstzweifel und Skepsis gegenüber der eigenen Weltkenntnis, wie sie in den frühen Briefen an de La Foye zum Ausdruck kamen, ebenfalls durch die Briefe.

Im Vergleich zu den Briefen an de La Foye hat sich im Briefwechsel mit der Ehefrau die Dialogsituation von einer Bruder-zu-Bruder- zu einer Vater-zu-Kind-Geste verschoben, damit sowohl dem tatsächlichen Altersunterschied der beiden Ehegatten Rechnung tragend als auch den Schreibstandards des 19. Jahrhunderts. Bedeutungsträchtige, in den Briefen an de La Foye uns begegnende Symbole finden sich auch in den Briefen der Greifswalder Reise wieder: das Motiv der einen Stube für zwei, ${ }^{30}$ des Siegelrings, ${ }^{31}$ ja selbst der für den Nordsternbund typischen Grußformel, die als „griechischer gelehrter Gruß ${ }^{\prime 32}$ eingesetzt wird. Diese Elemente schaffen eine Kontinuität im Briefhabitus.

Antonie fügte sich dem Erwartungshorizont als Ehefrau, als Mutter, als Briefschreiberin, indem sie erst abends, nach erledigtem Tagespensum, ${ }^{33}$ schrieb und dabei immer so müde war, dass sie kaum die Feder, ${ }^{34}$ ja nicht einmal die Augen offenhalten ${ }^{35}$ konnte. Auch kam sie den Erwartungen ihres Mannes als ungelehrte Frau entgegen („Du weißt ja, dass ich keine geübte Schreiberin bin “36) oder wurde am Schreiben gehindert, weil gerade geschneidert wurde. ${ }^{37}$ Ebenso berichtete sie von einer Predigt über Gehorsam und bekannte sich leidenschaftlich dazu. Oder sie gestand, sie liebe Chamisso mehr als er sie und freue sich darüber. ${ }^{38}$ Mit diesen, in den Briefen verstreuten Aussagen inszenierte sie sich selbst als Bilderbuchehefrau. Die Frage, ob dies von ihrer Seite als (wenn auch möglicherweise unbewusste) Strategie zur Eroberung eines Freiraums gedeutet werden könnte z. B. um den erotischen Träumen und zahlreichen Umarmungsphantasien Gehör zu verschaffen - sei hier dahingestellt. Vielmehr soll es darum gehen, was der Schriftsteller selbst daraus machte.

1804 hatte Chamisso seine Weltskepsis als erbitterte Unwissenheit und Unkenntnis der Welt inszeniert:

\footnotetext{
${ }^{29}$ Brief an Antonie vom 11. Juni 1823. Um die schmerzhaften Stiefel geht es auch im Brief vom 21. Juni 1823: „das gehen wird mit nur aus einem Umstand beschwerlich - daß nämlich meine Stiefel zu weit waren, der eine sehr schief getreten und mich gedrückt hatte".

${ }^{30}$ Brief von Antonie vom 10., 1..1 und 12, Juni 1823. Auch wenn das Zimmer des Ehepaars (im Gegensatz zum phantasierten gemeinsamen Zimmer mit de La Foye) zwei Betten hat, lässt es, wie es die Folgebriefe zeigen, reichlich Raum für erotische Phantasien zu.

${ }^{31}$ Brief von Antonie vom 14. und 15. Juni 1823.

32 Brief an Antonie vom 14. Juni 1823.

${ }^{33}$ Brief von Antonie vom 18. und 19. Juni 1823: „zugleich freue ich mich und danke Gott, wenn der Abend erst herangekommen ist, weil es wirklich die einzige Zeit ist, wo ich ungestört, mit Dir, mich unterhalten kann“.

${ }^{34}$ Brief von Antonie vom 14. und 15. Juni 1823.

${ }^{35}$ Brief von Antonie vom 4., 5., 6. und 7. Juli 1823, unter Datum des 6. Julis: „Das Schreiben wird mir jetzt des Abends recht sauer die Augen thun mir immer so weh, drum will ich Dir für heute gute Nacht sagen."

${ }^{36}$ Brief von Antonie vom 14. und 15. Juni 1823.

${ }^{37}$ Brief von Antonie vom 21. Juni 1823.

${ }^{38}$ Brief von Antonie vom 10., 12., 13. und 14. Juli 1823: „Du hast es mir auch schon zugestanden daß ich Dich mehr liebe als Du mich, ich bin auch mit der Anweisung, die wir Frauen erhalten haben, ganz zufrieden“.
} 
Ich mögte mit Fäusten mich schlagen! Ein Kerl von 24 Jahren, und nichts gethan, nichts erlebet, nichts genossen nichts erlitten, nichts geworden, nichts erworben, nichts, rein nichts, in dieser erbärmlichen erbärmlichen Welt ${ }^{39}$

In den Briefen an Antonie wiederum wurde mit Reife und Alter ${ }^{40}$ die Unfähigkeit zum Enthusiasmus in der Beobachtung der Welt thematisiert - und damit auch die Unfähigkeit zur "richtigen“ Beobachtung. So lässt sich folgende Aussage Chamissos an Antonie verstehen, geschrieben zu einem Zeitpunkt, an dem er noch hoffte, sie würde nach Greifswald reisen, um ihn auf seiner RügenExkursion zu begleiten:

Es ist mir sehr klar geworden, wie ich nur mit und durch dich Freude an meiner Rügner Reise haben könnte, daß ich wieder zum ersten Mal durch Deine Augen die See sehen dürfte, vom Stubenkammer aus in die dir noch soweite weite Welt hinaus schauen, Jung wieder werden in Dir, nichts gesehen haben, und wieder zum ersten Male sehen. ${ }^{41}$

Das Briefschreiben als Spaltung des Ichs zur Versenkung in eine andere Perspektive kommt bereits in Briefen an de La Foye vor: „kannst du dich in mich hinein denken “42, fragte Chamisso im Frühling 1812. Im Falle Antonies aber geht es nicht darum, durch sie zu denken, sondern, durch sie zu sehen. So wurde der traditionelle geschlechterspezifische Diskurs über weibliche Natürlichkeit vs. männliche Künstlichkeit in Formen der Weltwahrnehmung und -wiedergabe übersetzt. Es heißt im Anschluss daran, über das Barometer spottend:

Wenn nicht anders ist, werde ich hübsch gelehrt nach meinem Barometer sehen, und das Meer da drunten branden sehen - du würdest mir gewiß Lieder gemacht haben. ${ }^{43}$

Lieder gegen Barometer, Dichtung gegen Gelehrsamkeit? So eindeutig ist die Spaltung doch nicht. Das zeigt insbesondere die Funktion, die in diesem Briefwechsel Blumen zukommt.

Die Briefe an Antonie enthalten zahlreiche Hinweise auf das Blumeneinlegen. So schrieb Chamisso:

Könnte wohl Vater die Digitalis Purpurea im Logen Garten oder in einem anderen für Geld und gute Worte für 30 Exemplare auftreiben (die kleinsten sind die liebsten) du würdest sie einlegen. [...] - Für die Kornblume rechne ich auf euch. ${ }^{44}$

Die Antonie einbeziehende botanische Tätigkeit hielt während der gesamten Reise an. Antonie machte einen Ausflug nach Schöneberg, um Chamissos Informationen seinem Vorgesetzten im Botanischen Garten, Schlechtendal, mitzuteilen. ${ }^{45}$ Sie stritt sich sogar mit diesem darüber, wer von innen beiden die Kornblumen einlegen dürfe. ${ }^{46}$ Auch das Ende der Reise war mit dem Abschluss eines Blumenlebenszyklus verbunden. Eine Woche vor der Rückkehr schrieb Chamisso:

\footnotetext{
${ }^{39}$ Brief an de La Foye vom 20. September 1804. Noch 1806 heißt es: „Die Minuten sind von Gold so wichtig und fallen dahin, - eh ich mich umschaue hat es mit mir geendet" (Brief an de La Foye vom 9. Dezember 1806).

${ }^{40}$ Noch in einem Brief an de La Foye aus dem Jahr 1821 heißt es: „Heut bin ich 40 Jahre alt. Und du?“ (Brief an de La Foye vom 30. Januar 1821).

${ }^{41}$ Brief an Antonie vom 25. Juni 1823.

${ }^{42}$ Brief an de La Foye aus Genf oder Coppet, Frühling 1812.

${ }^{43}$ Brief an Antonie vom 25. Juni 1823.

${ }^{44}$ Brief an Antonie vom 28. Juni 1823. Vgl. ihre Antwort darauf im Brief vom 1., 2., 3. und 4. Juli 1823. Vgl. ebenso ihren Brief vom 22. und 23. Juni 1823: „Die beiden Pflanzen haben wir nicht vergessen.“

${ }^{45}$ Ihre Rolle als Botin wird in ihrem Brief vom 18. und 19. Juni 1823 angedeutet: „ich habe dem Schlechtendal Deinen letzten Brief den Dienstag auf dem Wege nach der Faueninsel herrauf gebracht."

${ }^{46} \mathrm{Vgl}$. Brief von Antonie vom 24., 25., 26. und 27. Juni 1823: „Heute morgen, mein lieber Adelbert, sind wir [...] nach Schönberg gegangen, [...] zu Schlechtendal, um zu fragen, ob er Deine Briefe bekommen habe den ich gleich gestern hinschickte, wir sagten ihm daß wir Dir Kornblumen hinter Schönberg hohlen wollten, er suchte es uns auszureden, weil er sie morgen selbst wollte einlegen, indeß lies ich es mir nicht nehmen, er ging mit uns und sagte mir weil die Exemplare so sehr viel zu groß sind und keine kleinen zu finden, ich möchte sie mir
} 
Du könntest immer die Pflanzen, die noch unter der Presse waren, in grossen Bogen heraus nehmen, damit ich freies Papier vorfände. Vielleicht komme ich, wenn das Wetter schön ist, gleich mit einer Heuernte heim. Man muß das Geschäft nicht versäumen, und diese Fahrt hat mich nun etwas verspätet $-{ }^{47}$

Zieht man noch den Briefwechsel mit de La Foye heran, lässt sich eine Metamorphose des floralen Themas bei Chamisso rekonstruieren. In der Zeit um 1810 waren Blumen noch bevorzugter Teil seiner Dichtung, nicht zuletzt unter dem Einfluss Helmina von Chézys. Ab 1811 wurden sie, aufgelistet, zu Forschungsgegenständen. Bereits 1814 schrieb Chamisso an de La Foye über sein Herbarium, dies sei:

mein Schatz und meine Lust - dass soll es sein und bleiben, lieber was würde aus mir, wenn mir das Heu zu widerstehen anfinge. ${ }^{48}$

1823 waren es Blumen, die die bereiste Gegend interessant machten. ${ }^{49}$ Als Dichtungsgegenstand waren sie nun unter der Feder des Botanikers depotenziert. Das waren sie allerdings auch als Teil von Liebesbeziehungen im Alltag. So schrieb Antonie:

Bitte, pflüke doch ja eine Blume für mich, aber ja pflüke sie mit der Absicht sie mir zu bringen, damit es mir ein Andenken sei, wie du auch in der Ferne Deiner Antonie denkst. ${ }^{50}$

In dieser Forderung klingt eine Bitte um Rückversicherung über den Stellenwert ihrer Beziehung, sozusagen eine Bitte um Liebeserklärung, an. Denn gibt es eine offensichtlichere Liebesgeste, als auf Reisen eine Blume für die Geliebte zu pflücken und diese mitzubringen? Die Aufforderung kann aber auch als eine Mahnung verstanden werden, der Natur ihren sensiblen Platz wiederzugeben: Blumen sind nicht nur Forschungsobjekte, sie sind mit Affekt besetzte Erinnerungsobjekte.

Tatsächlich ließ sich Chamisso im Laufe der Greifswalder und Rügener Reise immer mehr von Affekten durchdringen. Vom Schreibduktus her setzen sich die letzten Briefe von den vorhergehenden ab. Folgende Passage zeigt exemplarisch, wie das (Affekt-)Ich durch die Verbindung mit der Adressatin in der Situation des Briefschreibens mit Kraft hervorgehoben wird:

Deine Briefe machten so ein Teil meines Lebens aus. Es fällt jetzt aus - und doch nur einen Tag! Mag sein, aber ich empfinde es doch. Ich weiß nicht recht, was ich sage, Dich von unserem Volksfest zu unterhalten fehlt mir die Laune, ich habe noch manches zu kramen, und der Zeiger der Uhr steht nicht still. ich habe dir im Grunde nichts zu sagen, als was Du schon weißt - Ich liebe Dich, ich komme zu Dir. Ich bin Dein Vater, Dein Mann Dein Bräutigam, dein Freund und dein Geliebter. ${ }^{51}$

\section{3. „auch ist mein Heu nichts weniger als in Ordnung “52 - zur Ordnung der Dinge bei Chamisso}

nach den Bogen schneiden, das habe ich den gründlich befolgt, den Holunder wollte er mir nicht überlassen, er will Dir auch über alle diese Dinge Bericht erstatten. " Dass sie durchgehend mit Schlechtendal in Kontakt steht, lässt sich ihrem Brief vom 10., 11., 12. und 14. Juli entnehmen: „(Schlechtendal hat mir gestern gesagt er freue sich auch sehr auf deine Rükkunft)“.

${ }^{47}$ Brief an Antonie vom 12. Juli 1823.

${ }^{48}$ Brief an de La Foye vom Winter 1814. Vgl. auch schon im Juni oder Juli 1812: „Ich hatte sehr anspruchslos Pflanzen zu trocknen angefangen, - und nun zählt mein Herbarium bald tausend gattungen, und nun fängt es an mir als eine Habe mir zu erscheinen [...]".

${ }^{49}$ Zu Beginn der Reise heißt es: „Ein ekelhafteres Land giebt es wohl auf der Erde nicht wieder, Kein Pflänzlein in dieser grauen Wüstenei, die einen Botaniker erfreuen könnte.“ (Brief an Antonie vom 11. Juni 1823.)

${ }^{50}$ Brief von Antonie vom 7. Juli 1823.

${ }^{51}$ Brief an Antonie vom 12. Juli 1823. Besonders hervorgehoben seien hier die zahlreichen „ich“-Assonanzen.

${ }^{52}$ Ebd. 
Die Reisebriefe an den Freund bzw. an die Frau zeigen die Verwandlung vom jungen Dichter hin zum Naturwissenschaftler, der dann - der Ausblick auf die 1830er Jahre ist schon in den Briefen an Antonie angelegt - wieder zum Dichter finden wird. Bei aller Ähnlichkeit in Ton und Duktus änderte sich jedoch zwischen den Jugendbriefen und den Briefen aus den 1820er Jahren Einiges. Es sei hier die These aufgestellt, dass die Wende, auf die die Briefe bis 1812 zusteuern, deswegen eine Wende ist, weil Chamisso sich von da an zu einer gewissen Selbst- und Weltordnung bekannte.

\section{- Ordnung der Welt (Wissenschaft):}

Die Wendung zu einem naturwissenschaftlichen Studium wird von Chamisso nicht im Sinne einer Fachspezialisierung, sondern enzyklopädisch, als Ansatz zu einem Gesamtverständnis der Welt, konzipiert. 1812 stand nicht das bereits erkundete Pflanzenreich im Mittelpunkt des angestrebten Studiums, sondern das Tierreich. Die Ambition war eine ausdrücklich allgemeine:

diesen Winter freilich anatomie nebenbei Zoologie und Botanik, künftigen anatomia comparata physiologie und Botanik, mein Zweck ist eben nicht zu pracktizieren, ob ich gleich nach dem Doktorhut ringen werde, ich will alle Naturwissenschaften mehr oder weniger umfassen, und in einigen Jahren als ein gemachter Mann und ein Rechter Kerl vor mir stehen, der zu einer gelehrten Reise im allgemeinen, und zu einer grossen Unternehmung der Art, als tauglich sich darstellen könnte. ${ }^{53}$

Wenige Monate später fasste er seine eigene Entwicklung in Worten einer Spannung zwischen Phantastik und Skepsis mit folgenden Worten zusammen: „So stehts mit mir Adelf - Ich hebe mich von der Spekulation zu der Erfahrung[.] “54

Dieses Weltbild galt den gesamten menschlichen Verhältnisse. Nach erbitterten Betrachtungen über die französische Politik merkte Chamisso an:

ich habe mehr Freude an meinen Eingeweide Würmer die ich zuletzt auf die Muster zu bestimmen und in Ordnung zu bringen habe. ${ }^{55}$

Ordnung herzustellen war ebenfalls der Zweck des Reisens als Auseinandersetzung mit der wilden, nicht geordneten Natur: ${ }^{56}$ Sensible Erfahrungen (die Reise zu Fuß, die Erkundung Rügens „durch die Augen“ Antonies) und Beobachtungen machten dabei die Grundlage des Schreibens aus. ${ }^{57}$ In Greifswald ordnete die naturwissenschaftliche Tätigkeit nicht nur Naturphänomene, sondern auch soziale Verhältnisse. So schrieb Chamisso kurze Zeit nach seiner Ankunft:

Ich bin Prophet in seinem Lande, ich coursire als Weltumsegler Conversations Lexikons Mann, und Mann ohne Schatten. Die Professoren der Universität kommen mir entgegen. ${ }^{58}$

Dieser ordnende Zugang zur Natur war mit einem allgemein konservativen, aber manchmal doch offeneren Verständnis der Geschlechterrollen verbunden. So hieß es beispielsweise: „Ach ich

\footnotetext{
${ }^{53}$ Brief an de La Foye vom Herbst 1812.

${ }^{54}$ Brief an de La Foye von wahrscheinlich 1813.

${ }^{55}$ Brief an de La Foye vom März-Juni 1814.

${ }^{56}$ So etwa auch die Passivität in der Phase vor der Abreise zur Weltumseglung: „Lieber Freund ich liege auf meinem Heu in Erwartung des frischen Grasses lasse die welt gehn und ziehe einen fuß nach dem andern aus ihr heraus niemand und nichts fesselt mich recht mehr und ich glaube man mögte fast so ein abgekühltes Thier einen Philosophen nennen, ware nicht grade das erste was ich hasse und verachte die Philosophie -“ (Brief an de La Foye vom 23. Januar 1815).

57 "Sonnabends fange ich zu beobachten an und werde mehr Sachen zu schreiben haben“: Brief an Antonie vom 18. Juni 1823.

${ }^{58}$ Brief an Antonie vom 18. Juni 1823.
} 
wünschte ich hätte eine weibliche leidenschaftliche Liebe zur Wissenschaft. ${ }^{\text {"59 }}$ So leichte ließen sich weibliche Fantasie und männliche Skepsis doch nicht voneinander trennen.

\section{- Ordnung der Geschlechter}

Obwohl das o.g. Zitat einem Brief an de La Foye entnommen ist, findet sich Lob der weiblichen Weltwahrnehmung vor allem erwartungsgemäß in den Briefen an Antonie, allerdings eingebettet in einen traditionellen Diskurs der Rollenverteilung der Geschlechter.

In den außerehelichen Briefen hingegen wird dieser Diskurs hinterfragt. Der Briefwechsel mit de La Foye ist mit Liebesbeteuerungen übersät, die sich grundsätzlich im Rahmen des damaligen Briefduktus unter Männern bewegen. Dennoch fallen an einzelnen Stellen besonders ambigue Aussagen auf. ${ }^{60}$ Auch Transferphänomene sind zu finden, bezeichnenderweise in der Periode der inn affektiv überwältigenden Liebesbeziehung mit Helmina von Chézy. So etwa eine „wundersame ergreifende Scene":

Wir [= Chamisso und Helmina] waren mißmutig und es schlug eben nichts an - sie hub an von ihrer Liebe, von ihrem nagenden Kummer, mich nicht früher, vor 10 Jahren, wo sie mich (doch noch in mir selbst Schlummernden) wohl geahndet, und schon ganz zu mir sich hinneigt, mich sag ich damals nicht ganz gekannt, mich nicht ganz geliebt, mich nicht wie nun zu spät, nach einem verpfuschten nicht rein gebliebenen Leben erfaßt zu haben: da wäre es Zeit gewesen, - Zweifelte auch wieder, ob und wie ich sie liebte. Freundschaft, so etwas, nicht verstoßen wollen, lieben weil sie so unglücklich, weil ich so gut und mild: sie hielt traurend meine Hand - es schnitt mir ins Herz und ich sagte nun Gott weiß wie und mit welchem Tone: Ich liebe dich. Da preßte sie krampfhaft stumm trennen-los ihre Lippen auf meine Hand und ihr langer Kuß ging in eine Ohnmacht über aus der Ströme von Tränen über ihr verkläre leuchtenden Angesicht sie spät erhohlten da wär ihr gedanken gewesen: nun sterben, nun wußte sie es. ${ }^{61}$

Diese Geschlechterordnung wankt hier an zwei Stellen: erstens, als Chamissos eigene Stimme in dieser Schilderung bei den Worten "gott weiss wie und mit welchem Tone“ wackelig erscheint, und zweitens, weil der dieser Passage vorangehende Satz lautet: „Wir speisten selbander bei Grignon, hatten ein Kabinet wo ich noch einmal mir dir zusammen speisen will. ${ }^{\text {62 }}$

Ob nun eine Wiederholung der Liebeserklärungszene samt Ohnmacht mit de La Foye in der Hauptrolle auch mit den Phantasien des "guten, göttlichen Jungen ${ }^{463}$ vereinbar gewesen ist, lässt sich den Briefen nicht entnehmen. ${ }^{64}$ Aber es lässt sich beobachten, dass die generationelle Ordnung die Geschlechterrollen festigte, und dies schon bei der Nachricht der frühen Geburt des Kindes, das

\footnotetext{
${ }^{59}$ Brief an de La Foye von März-Juni 1814.

${ }^{60}$ In einem Brief, wo Chamisso von sexuellen Avancen eines Mannes berichtet, schließt er den Bericht mit der andeutungsvollen Frage: „bißt du auch schon so geliebt worden, haben auch Jünglinge deiner begehrt?“ (Brief an de La Foye vom 20. September 1804).

${ }^{61}$ Brief an de La Foye von Juli 1810.

62 Ebd.

${ }^{63}$ Vgl. z. B. im Brief vom 15. August 1804.

${ }^{64}$ In dieser Hinsicht mehrdeutige Stellen lassen sich häufiger dem Anfang des Briefwechsels entnehmen, etwa dem Brief an de La Foye von Ende Oktober 1804: „[...] wir müssen da sein, und schauen, und an uns selbst zehren, auf daß wir selber verwelken, und nicht Todt bringen dem Geliebten. und wenn wir einst nachdem die Tage der Köstlichen Jugend durchlitten die Bande schliessen die man auch noch wohl der Ehe zu nennen sich erkünet [...]".
} 
er mit Helmina gezeugt hatte. Die Geburt der legitimen Söhne nach der Eheschließung mit Antonie machte dies noch anschaulicher. ${ }^{65}$

Doch es wäre übertrieben, dies als ausschließlich biedermeierliche Fügung zur Gesellschaftsordnung zu lesen. Chamissos späterer Gedichtzyklus „Frauenliebe und -leben“ (1830) kann etwa genauso plausibel als Adaption der Geschlechterordnung gelesen werden, denn als Spiel mit dieser. In diesen Gedichten ist das lyrische Ich eine junge Braut (zuerst Geliebte, dann Verlobte, dann Braut, dann Ehefrau). So übernimmt Chamisso Antonies Rollen und ihre Stimme. Der Einfühlung in das weibliche Körpergefühl wird dabei im 7. Gedicht „An meinem Herzen an meiner Brust" bemerkenswert Ausdruck verliehen. Wiederholt auftretende Motive lassen sich auf Antonies Biographie zurückführen, so etwa die Schwesternschar im 5. Gedicht „Helft mir, Ihr Schwestern“, die bereits im 1. Gedicht mit der Erwähnung der "Schwesternspiele“ vorkommt. Auch die Heirat mit dem Ehepartner als eine in der Kindheit zurückliegende Phantasie (4. Gedicht) kann in Anlehnung an Antonies Biographie gelesen werden. Darüber hinaus weisen die Gedichte thematische Berührungspunkte mit dem Briefwechsel der Greifswalder Reise auf. Im ersten Gedicht ist dies der Fall bei der im Gedicht zentralen Thematik der Fokussierung der Welt durch den Blick des Ehepartners (wobei hier nicht der Mann durch die Augen der Frau sieht, sondern umgekehrt - eine Art Transposition von Chamissos Standpunkt in Antonies). Die Hierarchie der Demut der Frau vs. Verherrlichung des Mannes (2. und 5. Gedicht), das Motiv des Traums (3. und 4. Gedicht) und die erotischen Anspielungen (6. Gedicht) ziehen ebenfalls Linien, die eine Inspiration durch die Briefe der Greifswalder Reise suggerieren.

Das letzte, 8. Gedicht, „Nun hast Du mir den ersten Schmerz getan“, hebt sich von dieser Logik des Stimmentausches ab:

Ich zieh mich in mein Innres still zurück,

Der Schleier fällt,

Da hab ich dich und mein vergangnes Glück,

Du meine Welt! ${ }^{66}$

Hier sind beide Stimmen austauschbar: Der Tod ebnet die Ordnung der Geschlechter. Der lyrisierte Tod indessen führt den Dichter zurück zu sich selbst und veranschaulicht die Eitelkeit der weltlichen Verhältnisse. An diesem Beispiel zeigt sich, wie Chamisso nicht nur als Botaniker, sondern als Dichter seinen Briefwechsel mit Antonie führte.

\section{- Ordnung des Diskurses}

Die sich im Laufe der hier betrachteten Jahre wandelnde Welt- und Gesellschaftswahrnehmung lässt sich an Änderungen in Chamissos Schreibstil nachverfolgen. Die Entwicklung des Schreibduktus in den Briefen an de La Foye ist dabei von besonderem Interesse. In der Almanachsphase lyrisch angelegt, erreichten die Briefe in der Chézy-Phase ihren hyperbolischen Höhepunkt, danach wurden sie trockener. Der Ärger über die Coppet'sche Geselligkeit gab zwar Anlass zu einer Rückkehr zur Selbstbezüglichkeit, dennoch blieben die langen lyrischen Höhenflüge aus. Der Stil wurde knapper, nicht mehr von Wort- und Halbsatzwiederholungen geprägt. Mit den Pflanzenlisten, den botanischen Bemerkungen, dem Austauschen von Blumendoubletten erreichte der Briefwechsel peu à peu den Anstrich eines Gelehrtenbriefwechsels, bis er es realiter geworden war und ein anderer

\footnotetext{
${ }^{65}$ Der älteste Sohn wird übrigens im Briefwechsel mit Antonie „mein Camerad“ genannt, genauso wie es de La Foye gewesen war.

${ }^{66}$ Die Zitate aus dem Gedichtzyklus wurden Friedlaenders Ausgabe von Schumanns Liedern (Bd. I) entnommen, hier S. 105.
} 
Duktus vorherrschte. Die späteren Briefe an de La Foye blieben herzlich, aber sie waren dezidiert weniger affektiv besetzt und im Schreibstil prosaischer.

Die Briefe an Antonie ihrerseits zeigen, wie Chamisso trotz der weniger lyrischen Diktion, trotz der primär auf naturwissenschaftliche Beobachtungen hin gerichteten Schreibweise - Blumen einlegen, barometrische Messungen anstellen - und mithilfe von meist kurzen Satzbauelementen einen neuen Weg nach innen öffnet, zurück zu einem (lyrischen) Ich, das an den anderen - nicht immer, aber manchmal - wie an einen Teil des eigenen Ichs schreibt. ${ }^{67}$

Die Wechselbeziehungen zwischen Briefdiskurs, wissenschaftlichem Werk und Lyrik wurden hier am Beispiel der Behandlung der Reise als wissenschaftlicher Welt- und literarischer Selbsterkundung nachverfolgt. So konnten Parallelen zwischen Chamissos Jugend- und Erwachsenenjahren gezogen werden, die insbesondere den Gedichtzyklus Frauenliebe und -leben beleuchten.

Verglichen mit der Masse des aufbewahrten schriftlichen Materials zu Chamissos Werk kann das hier herangezogene Korpus nur beispielhaften Charakter haben. Aber diese intensive Auseinandersetzung mit den Handschriften hat gezeigt, dass es unerlässlich ist, um seine Schreibpraxis rekonstruieren zu können, diese zu leisten. Denn die in der hiesigen Analyse relevanten Aspekte hätten kaum anhand der redigierten Ausgaben Hitzigs, Palms oder Riegels durchgeführt werden können, welche Fragezeichen in Ausrufezeichen gewandelt und Pflanzenlisten aus dem Druck ausgeklammert haben.

Durch den Rückgriff auf heutige Briefeditionen werden solche neue Lektüren von Chamissos Schriftwerk möglich. Es ist zu hoffen, dass die Chamisso-Forschung sich die Grundlagen für diesen erneuten Zugang zum schriftstellerischen und botanischen Werk in der Form von Neueditionen schaffen wird.

\footnotetext{
${ }^{67}$ Hierzu vgl. auch den Brief an Fouqué vom 18. oder 19. Mai 1809: „Ich schicke mich an, mich an dich zu schicken [stellvertretend] für einen Brief, den ich Dir seit gar zu langer Zeit schuldig bin, zürne mir nicht, Guter, daß ich Dir nicht geschrieben, und daß ich annoch nicht komme." (Hitzig 1839, S. 228.)
} 


\section{Literaturverzeichnis}

Busch, Anna: Hitzig und Berlin. Zur Organisation von Literatur (1800-1840). Hannover, 2014.

Busch, Anna / Katins, Janine / Pravida, Dietmar: ,Polarsternbund (Nordsternbund)', in: Motschmann, Uta (Hg.): Handbuch der Berliner Vereine 1786-1815. Berlin, 2014, S. 16-26.

Chamisso, Adelbert von / Varnhagen, Karl August (Hg): Musenalmanach auf das Jahr 1804. Leipzig 1804.

Dies.: Musenalmanach auf das Jahr 1805. Zweiter Jahrgang. Berlin 1805.

Dies.: Musenalmanach auf das Jahr 1806. Dritter Jahrgang. Berlin 1806.

Dufraisse, Roger: ,Adelbert von Chamisso et Louis de La Foye. Contribution à l'étude des relations intellectuelles franco-allemandes à l'époque napoléonienne', in: Albrecht, Dieter u. a. (Hg.): Europa im Umbruch 1750-1850. München, 1995, S. 155-166;

Dufraisse, Roger: ,Un ami mal connu de Chamisso: Louis de la Foye', in: Chamisso. Actes des journées franco-allemandes des 30 et 31 mai 1981. Organisées par le Centre d'Études Argonnais, I'Académie Nationale de Reims et la Société d'Agriculture, Commerce, Sciences et Arts de la Marne. 1982, S. 6390.

Friedlaender, Max (Hg.): Robert Schumann, Lieder, Bd. I. Edition Peters Nr. 2383b.

Hitzig, Julius Eduard: Adelbert von Chamisso's Werke, 5. Band. Leben und Briefe, 1. Band. Leipzig 1839.

Palm, Friedrich (Hg.): Adelbert von Chamisso. Leben und Briefe, Bd. 5/6 von: Adelbert von Chamisso, Werke. Berlin, 1864.

Schmid, Günther: Chamisso als Naturforscher. Eine Bibliographie. Leipzig, 1942 